\title{
A parameter uniform difference scheme for the parameterized singularly perturbed problem with integral boundary condition
}

\section{Mustafa Kudu ${ }^{1 *}$}

"Correspondence:

muskud28@yahoo.com

1 Department of Mathematics,

Faculty of Arts and Sciences,

Erzincan University, Erzincan, Turkey

\begin{abstract}
We consider a uniform finite difference method on a Bakhvalov mesh to solve a quasilinear first order parameterized singularly perturbed problem with integral boundary conditions. Uniform first order error estimates in the discrete maximum norm have been established. Numerical results that demonstrate the sharpness of our theoretical analysis are presented.
\end{abstract}

MSC: 65L11;65L12;65L20

Keywords: Singular perturbation; Finite difference scheme; Uniform convergence; Parameterized problem; Bakhvalov mesh; Integral boundary condition

\section{Introduction}

Singularly perturbed differential equations are typically characterized by a small parameter $\varepsilon$ multiplying some or all of the highest order terms in the differential equations as normally boundary layers occur in their solutions. These equations play an important role in today's advanced scientific computations. Many mathematical models starting from fluid dynamics to the problems in mathematical biology are modeled by singularly perturbed problems. Typical examples include high Reynold's number flow in the fluid dynamics, heat transport problem, etc. For more details on singular perturbation, one can refer to the books [1-4] and the references therein. The numerical analysis of singular perturbation cases has always been far from trivial because of the boundary layer behavior of the solution. Such a problem undergoes rapid changes within very thin layers near the boundary or inside the problem domain [2,3]. It is well known that standard numerical methods for solving such problems are unstable and fail to give accurate results when the perturbation parameter is small. Therefore, it is important to develop suitable numerical methods to the problems, whose accuracies do not depend on the parameter value, i.e., methods that are convergent $\varepsilon$-uniformly. For the various approaches on the numerical solutions of differential equations with steep, continuous solutions, we may refer to the monographs $[1,4,5]$.

In this paper, we consider the following parameterized singular perturbation problem with integral boundary condition arising in many scientific applications [6, 7] (see also

(c) The Author(s) 2018. This article is distributed under the terms of the Creative Commons Attribution 4.0 International License (http://creativecommons.org/licenses/by/4.0/), which permits unrestricted use, distribution, and reproduction in any medium, provided you give appropriate credit to the original author(s) and the source, provide a link to the Creative Commons license, and indicate if changes were made. 
references therein):

$$
\begin{aligned}
& \varepsilon u^{\prime}+f(t, u, \lambda)=0, \quad t \in \Omega=(0, T), T>0, \\
& u(0)+\int_{0}^{T} c(s) u(s) d s=A, \\
& u(T)=B,
\end{aligned}
$$

where $\varepsilon \in(0,1]$ is the perturbation parameter, $\lambda$ is known as the control parameter, $A$ and $B$ are given constants. The functions $c(t) \geq 0$ and $f(t, u, \lambda)$ are assumed to be sufficiently continuously differentiable for our purpose in $\bar{\Omega}=\Omega \cup\{t=0\}$ and $\Omega \times \mathbb{R}^{2}$, respectively, and moreover

$$
\begin{aligned}
& 0<\alpha \leq \frac{\partial f}{\partial u} \leq a^{*}<\infty, \\
& 0<m_{1} \leq\left|\frac{\partial f}{\partial \lambda}\right| \leq M_{1}<\infty .
\end{aligned}
$$

By a solution of (1)-(3) we mean $\{u(t), \lambda\} \in C^{1}[0, T] \times \mathbb{R}$, for which problem (1)-(3) is satisfied. Under these assumptions, problem (1)-(3) has a unique solution $u(t)$. For $\varepsilon \ll 1$, the function $u(t)$ has in general a boundary layer of width $O(\varepsilon)$ near $t=0$ (see [8-10]).

Parameterized boundary value problems have been considered by many researchers for many years. Such problems arise in physical chemistry and physics, describing the exothermic and isothermal chemical reactions, the steady-state temperature distributions, the oscillation of a mass attached by two springs leading to a differential equation with a parameter $[5,11,12]$. An overview of some existence and uniqueness results and applications of parameterized equations may be obtained, for example, in [11-14] (see also references therein). In $[11,12,14,15]$, the authors have also considered some approximating aspects of this kind of problems. But in the above-mentioned papers, algorithms are only concerned with the regular cases (i.e., when the boundary layers are absent). In recent years, many researchers presented the numerical methods for the singular perturbation cases of parameterized problems. Uniform convergent finite-difference schemes for solving parameterized singularly perturbed two-point boundary value problems have been considered in $[8,10,15-22]$ (see also references therein). In $[8,10,16,17,19,20]$ authors used the boundary layer technique for solving an analogous problem. A methodology based on the homotopy analysis technique to approximate the analytic solution was investigated in $[15,21,22]$.

Also it is well known that nonlinear differential equations with integral boundary conditions have been used in description of many phenomena in the applied sciences, e.g., heat conduction, chemical engineering, underground water flow, and so on [23-25]. Therefore, boundary value problems involving integral boundary conditions have been studied by many authors $[6,7,26-31]$ (see also references therein). Some approximating aspects of this kind of problems in the regular cases, i.e., in the absence of layers, were investigated in $[7,26,32]$. In recent years, many researchers have considered the singularly perturbed cases for these problems. In [9] authors developed a finite difference scheme on Shishkin mesh for a problem with integral boundary conditions and proved that the method is nearly first order convergent except for a logarithmic factor. A hybrid scheme, which is 
second order convergent on Shishkin mesh, was discussed in [30] (see also [20,33]). For the numerical methods concerning second order singularly perturbed differential equations with integral boundary conditions, one can see, e.g., [28, 29, 31].

In this paper, as far as we know, the numerical solution of the singularly perturbed boundary value problem containing both control parameter and integral condition is first being considered. For the numerical solution of such problems, a specific approach is required to construct the appropriate difference scheme and examining the error analysis. The scheme is constructed by the method of integral identities with the use of appropriate quadrature rules with the remainder terms in integral form. The aim here is to construct an $\varepsilon$-uniformly numerical method which gives $\varepsilon$-uniformly convergent numerical approximations to solve problem (1)-(3). For this, we use a finite difference scheme on a Bakhvalov mesh which is dense in the initial layer. The Bakhvalov mesh is dependent on $\varepsilon$ and mesh points have to be condensed in a neighborhood of $t=0$ in order to resolve the initial layer. In the Bakhvalov mesh, basically half of the mesh points are concentrated in $O(\varepsilon|\ln \varepsilon|)$ neighborhood of the point $t=0$ and the remaining half forms a uniform mesh on the rest of $[0, T]$ (see $[2,4,10,30,32,34,35])$. We show that the proposed scheme is uniformly convergent in the discrete maximum norm accuracy of $O\left(N^{-1}\right)$ on Bakhvalov meshes. Note that, in [10], the first order convergent difference scheme in Bakhvalov type mesh under the first type boundary conditions for equation (1.1) was presented. Also, in the above-mentioned work [9] that includes integral boundary condition, while conditions (2.1) and (4.8) are generally provided for sufficiently small values of $\varepsilon$, as the integral boundary condition of our work is more general, and the convergence is uniform for both small and moderate values of perturbation parameter $\varepsilon$.

The paper is organized as follows. In Sect. 2, the difference scheme constructed on the non-uniform mesh for the numerical solution (1)-(3) is presented and graded mesh is introduced. The uniform convergence of the difference scheme is investigated and error of the difference scheme is evaluated in Sect. 3. Finally, in Sect. 4 some numerical results are presented to confirm the theoretical analysis. The paper ends with conclusions.

Henceforth, $C$ and $c$ denote the generic positive constants independent of both the perturbation parameter $\varepsilon$ and the mesh parameter $N$. Such a subscripted constant is fixed. We also will use $\|g\|_{\infty}=\max _{0 \leq t \leq T}|g(t)|$ for any $g \in C[0, T]$.

\section{The finite difference scheme}

To construct the numerical method and for convergence analysis, we need the asymptotic estimates for the differential solution $\{u(t), \lambda\}$.

Lemma 2.1 The solution $\{u(t), \lambda\}$ of problem (1)-(3) satisfies the following bounds:

$$
\begin{aligned}
& |\lambda| \leq c_{0}, \\
& \|u\|_{\infty} \leq c_{1},
\end{aligned}
$$

where

$$
\begin{aligned}
& c_{0}=m_{1}^{-1}\left\{\frac{\alpha|A|}{e^{\alpha T}-1}+\frac{|B| a^{*}\left(1-\|c\|_{\infty} T\right)}{m_{1}\left(e^{a^{*} T}-1\right)}+\|F\|_{\infty}\right\}, \\
& F(t)=f(t, 0,0),
\end{aligned}
$$




$$
c_{1}=A+\alpha^{-1}\left(1+\|c\|_{\infty} T\right)\left(\|F\|_{\infty}+M_{1} c_{0}\right)
$$

and

$$
\left|u^{\prime}(t)\right| \leq C\left(1+\frac{1}{\varepsilon} e^{-\frac{\alpha t}{\varepsilon}}\right), \quad t \in[0, T]
$$

provided $a \in C^{1}[0, T]$ and $\left|\frac{\partial f}{\partial t}\right| \leq C$ for $t \in[0, T]$.

Proof One can prove this result following the method given in [9], Lemma 2.1, and in [10], Lemma 2.1.

Let $\omega_{N}$ be any non-uniform mesh on $\Omega$ :

$$
\omega_{N}=\left\{0<t_{1}<t_{2}<\cdots<t_{N-1}<t_{N}=T\right\}
$$

and $\bar{\omega}_{N}=\omega_{N} \cup\{t=0\}$. For each $i \geq 1$, we set the step size $h_{i}=t_{i}-t_{i-1}$. To simplify the notation, we set $g_{i}=g\left(t_{i}\right)$ for any function $g(t)$, while $g_{i}^{N}$ denotes an approximation of $g(t)$ at $t_{i}$. For any mesh function $\left\{w_{i}\right\}$ defined on $\bar{\omega}_{N}$, we use

$$
\begin{aligned}
w_{\bar{t}, i} & =\left(w_{i}-w_{i-1}\right) / h_{i}, \\
\|w\|_{\infty} & \equiv\|w\|_{\infty, \bar{\omega}_{N}}:=\max _{0 \leq i \leq N}\left|w_{i}\right| .
\end{aligned}
$$

To obtain approximation for (1), we integrate (1) over $\left(t_{i-1}, t_{i}\right)$ :

$$
\varepsilon u_{\bar{t}, i}+h_{i}^{-1} \int_{t_{i-1}}^{t_{i}} f(t, u(t), \lambda) d t=0, \quad 1 \leq i \leq N
$$

which yields the relation

$$
\varepsilon u_{\bar{t}, i}+f\left(t_{i}, u_{i}, \lambda\right)+R_{i}=0, \quad 1 \leq i \leq N
$$

with local truncation error

$$
R_{i}=-h_{i}^{-1} \int_{t_{i-1}}^{t_{i}}\left(t-t_{i-1}\right) \frac{d}{d t} f(t, u(t), \lambda) d t .
$$

To define an approximation for the boundary condition (2), here we use the composite right-hand side rectangle rule:

$$
u(0)+\int_{0}^{T} c(s) u(s) d s=u_{0}+\sum_{i=1}^{N} h_{i} c_{i} u_{i}+r
$$

with remainder term

$$
r=-\sum_{i=1}^{N} \int_{t_{i-1}}^{t_{i}}\left(t-t_{i-1}\right) \frac{d}{d t}(c(t) u(t)) d t
$$


Consequently,

$$
u_{0}+\sum_{i=1}^{N} h_{i} c_{i} u_{i}+r=A .
$$

Neglecting $R_{i}$ and $r$ in (7) and (10), we propose the following difference scheme for approximating (1)-(3):

$$
\begin{aligned}
& \varepsilon u_{\bar{t}, i}^{N}+f\left(t_{i}, u_{i}^{N}, \lambda^{N}\right)=0, \quad 1 \leq i \leq N, \\
& u_{0}^{N}+\sum_{i=1}^{N} h_{i} c_{i} u_{i}^{N}=A, \\
& u_{N}^{N}=B .
\end{aligned}
$$

For the difference scheme (11)-(13) to be $\varepsilon$-uniformly convergent, we will use the B-mesh. For an even number $N$, the B-mesh takes $N / 2+1$ points in the interval $[0, \sigma]$ and also $N / 2+1$ points in the interval $[\sigma, T]$, where the transition point $\sigma$, which separates the fine and coarse portions of the mesh, is obtained by taking

$$
\sigma=\min \left\{\frac{T}{2}, \alpha^{-1} \varepsilon|\ln \varepsilon|\right\} .
$$

In practice one usually has $\sigma \ll T$, so the mesh is fine on $[0, \sigma]$ and coarse on $[\sigma, T]$. We shall consider a mesh $\omega_{N}$ which is equidistant in $[\sigma, T]$ but graded in $[0, \sigma]$ by a logarithmic mesh generating function. The corresponding mesh points are

$$
\begin{aligned}
& t_{i} \in[0, \sigma]: t_{i}= \begin{cases}-\alpha^{-1} \varepsilon \ln \left[1-(1-\varepsilon) \frac{2 i}{N}\right], & \text { if } \sigma<T / 2, \\
-\alpha^{-1} \varepsilon \ln \left[1-\left(1-\exp \left(-\frac{\alpha T}{2 \varepsilon}\right)\right) \frac{2 i}{N}\right], & \text { if } \sigma=T / 2, i=0, \ldots, N / 2,\end{cases} \\
& t_{i} \in[\sigma, T]: t_{i}=\sigma+(i-N / 2) h, \quad i=N / 2+1, \ldots, N, h=2(T-\sigma) / N .
\end{aligned}
$$

In the rest of the paper we only consider B-mesh defined by (14)-(16).

\section{Uniform convergence and error estimates}

To investigate the convergence of the method, note that the error functions $z_{i}^{N}=u_{i}^{N}-u_{i}$, $0 \leq i \leq N, \mu^{N}=\lambda^{N}-\lambda$ are the solution of the discrete problem

$$
\begin{aligned}
& \varepsilon z_{\bar{t}, i}^{N}+f\left(t_{i}, u_{i}^{N}, \lambda^{N}\right)-f\left(t_{i}, u_{i}, \lambda\right)=R_{i}, \quad 1 \leq i \leq N, \\
& z_{0}^{N}+\sum_{i=1}^{N} h_{i} c_{i} z_{i}^{N}-r=0, \\
& z_{N}^{N}=0,
\end{aligned}
$$

where the truncation errors $R_{i}$ and $r$ are given by (8) and (9), respectively.

Before estimating errors of the approximate solution, we need the known equalities for the first order difference equation, namely, the solution of

$$
y_{i}=q_{i} y_{i-1}+\varphi_{i}, \quad 1 \leq i \leq N
$$


can be expressed in the following forms:

$$
y_{i}=y_{0} Q_{i}+\sum_{k=1}^{i} \varphi_{k} Q_{i-k}
$$

or

$$
y_{i}=y_{N} Q_{N-i}^{-1}-\sum_{k=i+1}^{N} \varphi_{k} Q_{k-i}^{-1} \text {, }
$$

where

$$
Q_{i-k}= \begin{cases}1, & k=i, \\ \prod_{\ell=k+1}^{i} q_{\ell}, & 1 \leq k \leq i-1 .\end{cases}
$$

Relations (20) and (21) can be easily verified by induction in $i$.

Lemma 3.1 For the solution of (17)-(19), the following estimates hold:

$$
\begin{aligned}
& \left|\mu^{N}\right| \leq C|r|, \\
& \left|z_{0}^{N}\right| \leq|r|+\|c\|_{\infty} T B_{N}\left(\left|\mu^{N}\right| M_{1}+\|R\|_{\infty}\right), \\
& \left|z_{i}^{N}\right| \leq\left|z_{0}^{N}\right|+\alpha^{-1}\left(M_{1}\left|\mu^{N}\right|+\|R\|_{\infty}\right), \quad 1 \leq i \leq N-1,
\end{aligned}
$$

where

$$
\begin{aligned}
& B_{N}=\sum_{\ell=1}^{N} \frac{h_{\ell}}{\varepsilon+a_{\ell} h_{\ell}} Q_{N-\ell}, \\
& Q_{N-\ell}= \begin{cases}1, & \text { for } \ell=N, \\
\prod_{s=\ell+1}^{N} \frac{\varepsilon}{\varepsilon+a_{s} h_{s}}, & \text { for } 1 \leq \ell \leq N-1 .\end{cases}
\end{aligned}
$$

Proof Equation (17) can be rewritten as

$$
\varepsilon z_{\bar{t}, i}^{N}+a_{i} z_{i}^{N}=b_{i} \mu^{N}+R_{i}, \quad 1 \leq i \leq N-1,
$$

with

$$
\begin{aligned}
& a_{i}=\frac{\partial f}{\partial u}\left(t_{i}, u_{i}+\gamma z_{i}^{N}, \lambda+\gamma \mu^{N}\right), \\
& b_{i}=-\frac{\partial f}{\partial \lambda}\left(t_{i}, u_{i}+\gamma z_{i}^{N}, \lambda+\gamma \mu^{N}\right), \quad 0<\gamma<1 .
\end{aligned}
$$

From (25) we have

$$
z_{i}^{N}=\frac{\varepsilon}{\varepsilon+a_{i} h_{i}} z_{i-1}^{N}+\mu^{N} \frac{h_{i} b_{i}}{\varepsilon+a_{i} h_{i}}+\frac{h_{i} R_{i}}{\varepsilon+a_{i} h_{i}} .
$$


Solving the first-order difference equation with respect to $z_{i}^{N}$ by using (21) and setting the boundary condition (19), we get

$$
z_{i}^{N}=-\mu^{N} \sum_{k=i+1}^{N} \frac{h_{k} b_{k}}{\varepsilon+a_{k} h_{k}} Q_{k-i}^{-1}-\sum_{k=i+1}^{N} \frac{h_{k} R_{k}}{\varepsilon+a_{k} h_{k}} Q_{k-i}^{-1} .
$$

Taking into consideration in (26) the integral boundary condition (18), we have

$$
\begin{aligned}
\mu^{N}= & \frac{r}{\sum_{k=1}^{N} \frac{h_{k} b_{k}}{\varepsilon+a_{k} h_{k}} Q_{k}^{-1}+\sum_{k=1}^{N} h_{k} c_{k} \sum_{s=k+1}^{N} \frac{h_{s} b_{s}}{\varepsilon+a_{s} h_{s}} Q_{s-k}^{-1}} \\
& +\frac{\sum_{k=1}^{N} \frac{h_{k} R_{k}}{\varepsilon+a_{k} h_{k}} Q_{k}^{-1}+\sum_{k=1}^{N} h_{k} c_{k} \sum_{s=k+1}^{N} \frac{h_{s} R_{s}}{\varepsilon+a_{s} h_{s}} Q_{s-k}^{-1}}{\sum_{k=1}^{N} \frac{h_{k} b_{k}}{\varepsilon+a_{k} h_{k}} Q_{k}^{-1}+\sum_{k=1}^{N} h_{k} c_{k} \sum_{s=k+1}^{N} \frac{h_{s} b_{s}}{\varepsilon+a_{s} h_{s}} Q_{s-k}^{-1}} .
\end{aligned}
$$

Now, we estimate separately the terms on the right-hand side of equality (27). For the first term, we have

$$
\begin{aligned}
& \left|\frac{r}{\sum_{k=1}^{N} \frac{h_{k} b_{k}}{\varepsilon+a_{k} h_{k}} Q_{k}^{-1}+\sum_{k=1}^{N} h_{k} c_{k} \sum_{s=k+1}^{N} \frac{h_{s} b_{s}}{\varepsilon+a_{s} h_{s}} Q_{s-k}^{-1}}\right| \\
& \quad \leq \frac{|r|}{m_{1} \sum_{k=1}^{N} \frac{h_{k}}{\varepsilon+a_{k} h_{k}} Q_{k}^{-1}} \leq \frac{a^{*}|r|}{m_{1} \rho_{*} \sum_{k=1}^{N}\left(1+\rho_{*}\right)^{k-1}}=\frac{a^{*}|r|}{m_{1}\left[\left(1+\rho_{*}\right)^{N}-1\right]},
\end{aligned}
$$

here $\rho_{k}=a_{k} h_{k} / \varepsilon$ and $\rho_{*}=\min \rho_{k}$. Therefore, it is not hard to see that

$$
\left|\frac{r}{\sum_{k=1}^{N} \frac{h_{k} b_{k}}{\varepsilon+a_{k} h_{k}} Q_{k}^{-1}+\sum_{k=1}^{N} h_{k} c_{k} \sum_{s=k+1}^{N} \frac{h_{s} b_{s}}{\varepsilon+a_{s} h_{s}} Q_{s-k}^{-1}}\right| \leq C|r| .
$$

Next, evidently

$$
\left|\frac{\sum_{k=1}^{N} \frac{h_{k} R_{k}}{\varepsilon+a_{k} h_{k}} Q_{k}^{-1}+\sum_{k=1}^{N} h_{k} c_{k} \sum_{s=k+1}^{N} \frac{h_{s} R_{s}}{\varepsilon+a_{s} h_{s}} Q_{s-k}^{-1}}{\sum_{k=1}^{N} \frac{h_{k} b_{k}}{\varepsilon+a_{k} h_{k}} Q_{k}^{-1}+\sum_{k=1}^{N} h_{k} c_{k} \sum_{s=k+1}^{N} \frac{h_{s} b_{s}}{\varepsilon+a_{s} h_{s}} Q_{s-k}^{-1}}\right| \leq m_{1}^{-1}\|R\|_{\infty} .
$$

After taking into consideration (28) and (29) in (27), we arrive at (22). Now, we need to estimate $z_{0}$. From (26), by using (20) we have

$$
z_{i}^{N}=z_{0}^{N} Q_{i}+\mu^{N} \sum_{k=1}^{i} \frac{h_{k} b_{k}}{\varepsilon+a_{k} h_{k}} Q_{i-k}+\sum_{k=1}^{i} \frac{h_{k} R_{k}}{\varepsilon+a_{k} h_{k}} Q_{i-k} .
$$

From here, by virtue of (18) it follows that

$$
z_{0}^{N}=\frac{r-\sum_{k=1}^{N} h_{k} c_{k}\left(\mu^{N} \sum_{\ell=1}^{k} \frac{h_{\ell} b_{\ell}}{\varepsilon+a_{\ell} h_{\ell}} Q_{k-\ell}+\sum_{\ell=1}^{k} \frac{h_{\ell} R_{\ell}}{\varepsilon+a_{\ell} h_{k}} Q_{k-\ell}\right)}{1+\sum_{k=1}^{N} h_{k} c_{k} Q_{k}} .
$$

Thereby

$$
\left|z_{0}^{N}\right| \leq|r|+\|c\|_{\infty} T\left\{\left|\mu^{N}\right| \sum_{\ell=1}^{N} \frac{h_{\ell}\left|b_{\ell}\right|}{\varepsilon+a_{\ell} h_{k}} Q_{N-\ell}+\sum_{\ell=1}^{N} \frac{h_{\ell}\left|R_{\ell}\right|}{\varepsilon+a_{\ell} h_{k}} Q_{k-\ell}\right\}
$$




$$
\leq|r|+\|c\|_{\infty} T\left\{M_{1}\left|\mu^{N}\right|+\|R\|_{\infty}\right\} \sum_{\ell=1}^{N} \frac{h_{\ell}}{\varepsilon+a_{\ell} h_{\ell}} Q_{N-\ell}
$$

which implies validity of (23).

Finally, applying the maximum principle for the difference operator $L^{N} z_{i}^{N}:=\varepsilon z_{\bar{t}, i}^{N}+a_{i} z_{i}^{N}$, $1 \leq i \leq N$, to Eq. (25) immediately leads to (24).

Lemma 3.2 The error functions $R$ and $r$ satisfy

$$
\begin{aligned}
& \|R\|_{\infty, \omega_{N}} \leq C N^{-1}, \\
& |r| \leq C N^{-1} .
\end{aligned}
$$

Proof We first give proof for (30). From explicit expression (8) for $R_{i}$, on an arbitrary mesh we have

$$
\begin{aligned}
\left|R_{i}\right| & \leq h_{i}^{-1} \int_{t_{i-1}}^{t_{i}}\left(t-t_{i-1}\right)\left|\frac{\partial f}{\partial t}(t, u(t), \lambda)+\frac{\partial f}{\partial u}(t, u(t), \lambda) u^{\prime}(t)\right| d t \\
& \leq C h_{i}^{-1} \int_{t_{i-1}}^{t_{i}}\left(t-t_{i-1}\right)\left(1+\left|u^{\prime}(t)\right|\right) d t, \quad 1 \leq i \leq N .
\end{aligned}
$$

This inequality together with (6) enables us to write

$$
\left|R_{i}\right| \leq C\left\{h_{i}^{-1}+h_{i}^{-1} \varepsilon^{-1} \int_{t_{i-1}}^{t_{i}}\left(t-t_{i-1}\right) e^{-\alpha t / \varepsilon} d t\right\}, \quad 1 \leq i \leq N .
$$

$R_{i}$ is estimated on $[0, \sigma]$ and $[\sigma, T]$ separately. We consider first the case $\alpha^{-1} \varepsilon|\ln \varepsilon|<T / 2$, and so $\sigma=\alpha^{-1} \varepsilon|\ln \varepsilon|$. In $[\sigma, T]$, which is outside the layer $\left|u^{\prime}(t)\right| \leq C$ (or $\left.\varepsilon^{-1} e^{-\alpha x / \varepsilon} \leq 1\right)$ by (6) and $h_{i}=h$. Hereby, from (32) we get

$$
\left|R_{i}\right| \leq 2 C(T-\sigma) N^{-1}, \quad i=N / 2+1, \ldots, N .
$$

On the other hand, in the layer region $[0, \sigma]$, by (6), inequality (32) becomes

$$
\left|R_{i}\right| \leq C\left\{h_{i}+\alpha^{-1}\left(e^{-\frac{\alpha t_{i-1}}{\varepsilon}}+e^{-\frac{\alpha t_{i}}{\varepsilon}}\right)\right\}, \quad i=1, \ldots, N / 2 .
$$

Since

$$
\begin{aligned}
h_{i} & =t_{i}-t_{i-1}=\alpha^{-1} \varepsilon\left\{-\ln \left[1-(1-\varepsilon) \frac{2 i}{N}\right]+\ln \left[1-(1-\varepsilon) \frac{2(i-1)}{N}\right]\right\} \\
& \leq 2 \alpha^{-1}(1-\varepsilon) N^{-1}
\end{aligned}
$$

and

$$
e^{-\frac{\alpha t_{i-1}}{\varepsilon}}+e^{-\frac{\alpha t_{i}}{\varepsilon}}=2(1-\varepsilon) N^{-1},
$$

it then follows from (34) that

$$
\left|R_{i}\right| \leq 4 \alpha^{-1} C N^{-1}, \quad i=1, \ldots, N / 2 .
$$


Now consider the case $\sigma=T / 2$ and so $\sigma=\alpha^{-1} \varepsilon|\ln \varepsilon|$. Therefore, for $t_{i} \in[0, \sigma]$ with (15), we deal as in the case of the mesh region above. For $t_{i} \in(\sigma, T]$, since $h=2(T-\sigma) / N=T / N$,

$$
\max _{N / 2<i \leq N} \int_{t_{i-1}}^{t_{i}} \frac{1}{\varepsilon} e^{-\frac{\alpha t}{\varepsilon}} d t \leq h \frac{1}{\varepsilon} e^{-\frac{\alpha T}{2 \varepsilon}} \leq \frac{2 e^{-1}}{\alpha T} h=2 e^{-1} \alpha^{-1} C N^{-1},
$$

it follows from (32) that

$$
\left|R_{i}\right| \leq C\left(T+\frac{2}{e \alpha}\right) N^{-1}, \quad i=N / 2+1, \ldots, N
$$

and this together with (36) gives the bound

$$
\left|R_{i}\right| \leq C N^{-1}
$$

Inequalities (33), (35), and (36) finish the proof of (30).

Finally, we estimate the remainder term $r$. From the explicit expression (9) we obtain

$$
|r| \leq \sum_{i=1}^{N} \int_{t_{i-1}}^{t_{i}} c(t)\left|t-t_{i-1}\right|\left|u^{\prime}(t)\right| d t, \quad 1 \leq i \leq N .
$$

This inequality together with (6) reduces to

$$
|r| \leq\|c\|_{\infty} C \sum_{i=1}^{N} h_{i} \int_{t_{i-1}}^{t_{i}}\left(1+\frac{1}{\varepsilon} e^{-\frac{\alpha t}{\varepsilon}}\right) d t, \quad 1 \leq i \leq N .
$$

From (37), the validity of (31) follows:

$$
\begin{aligned}
|r| & \leq C \sum_{i=1}^{N / 2} h_{i} \int_{t_{i-1}}^{t_{i}}\left(1+\frac{1}{\varepsilon} e^{-\frac{\alpha t}{\varepsilon}}\right) d t+C \sum_{i=N / 2+1}^{N} h \int_{t_{i-1}}^{t_{i}}\left(1+\frac{1}{\varepsilon} e^{-\frac{\alpha t}{\varepsilon}}\right) d t \\
& \leq C\left\{N^{-1}+\sum_{i=1}^{N / 2} h_{i} \int_{t_{i-1}}^{t_{i}}\left(1+\frac{1}{\varepsilon} e^{-\frac{\alpha t}{\varepsilon}}\right) d t\right\} \\
& \leq C\left\{N^{-1}+\alpha^{-1} \sum_{i=1}^{N / 2} h_{i}\left(e^{-\frac{\alpha t_{i-1}}{\varepsilon}}+e^{-\frac{\alpha t_{i}}{\varepsilon}}\right)\right\} \\
& \leq C\left(N^{-1}+\sum_{i=1}^{N / 2} h_{i}\right) \leq C N^{-1} .
\end{aligned}
$$

Theorem 3.1 Let $\{u(t), \lambda\}$ and $\left\{u_{i}^{N}, \lambda^{N}\right\}$ be the exact solution and the discrete solution on $\bar{\omega}_{N}$, respectively. Then the following estimates hold:

$$
\begin{aligned}
& \left|\lambda-\lambda^{N}\right| \leq C N^{-1}, \\
& \left\|u-u^{N}\right\|_{\infty, \varpi_{N}} \leq C N^{-1} .
\end{aligned}
$$

Proof This follows immediately by combining the previous lemmas. 


\section{Algorithm and numerical results}

The results of the numerical experiment are presented in this section, which confirms the theoretical bounds established in the previous section.

(a) We solve the nonlinear problem (11)-(13) using the following quasilinearization technique:

$$
\begin{aligned}
& \lambda^{(n)}=\lambda^{(n-1)}-\frac{\left(B-u_{N-1}^{(n-1)}\right) \rho_{N}^{-1}+f\left(T, B, \lambda^{(n-1)}\right)}{\partial f / \partial \lambda\left(T, B, \lambda^{(n-1)}\right)}, \\
& u_{0}^{(n)}=A-c_{N} h_{N} B-\sum_{i=1}^{N-1} h_{i} b_{i} u_{i}^{(n-1)}, \\
& u_{i}^{(n)}=u_{i}^{(n-1)}-\frac{\left(u_{i}^{(n-1)}-u_{i-1}^{(n)}\right) \rho_{i}^{-1}+f\left(t_{i}, u_{i}^{(n-1)}, \lambda^{(n)}\right)}{\partial f / \partial u\left(t_{i}, u_{i}^{(n-1)}, \lambda^{(n)}\right)+\rho_{i}^{-1}}, \quad n=1,2, \ldots,
\end{aligned}
$$

where $\rho_{i}=h_{i} / \varepsilon ; \lambda^{(0)}$ and $u_{i}^{(0)}(1 \leq i \leq N-1)$ are the initial iterations given.

(b) Consider the test problem:

$$
\begin{aligned}
& \varepsilon u^{\prime}+2 u-e^{-u}+t^{2}+\lambda+\tanh (\lambda+t)=0, \quad 0<t<1, \\
& u(0)+\frac{1}{4} \int_{0}^{1} e^{-s} u(s) d s=1, \\
& u(1)=0 .
\end{aligned}
$$

The exact solution of our test problem is not available. Therefore we use the double mesh principle to estimate the errors and to compute the experimental rates of convergence. The error estimates obtained in this way are denoted by

$$
e_{u}^{\varepsilon, N}=\max _{\omega_{N}}\left|u^{\varepsilon, N}-u^{\varepsilon, 2 N}\right|, e_{\lambda}^{\varepsilon, N}=\left|\lambda^{\varepsilon, N}-\lambda^{\varepsilon, 2 N}\right|
$$

The corresponding rates of convergence are calculated by

$$
p_{u}^{\varepsilon, N}=\ln \left(e_{u}^{\varepsilon, N} / e_{u}^{\varepsilon, 2 N}\right) / \ln 2
$$

for $u$, and

$$
p_{\lambda}^{\varepsilon, N}=\ln \left(e_{\lambda}^{\varepsilon, N} / e_{\lambda}^{\varepsilon, 2 N}\right) / \ln 2
$$

for $\lambda$.

In the computations in this section we take $\alpha=2$. The initial guess in the iteration process is taken as $u_{i}^{(0)}=1-t_{i}^{2}, \lambda^{(0)}=-0.4$ and the stopping criterion is

$$
\max _{i}\left|u_{i}^{(n)}-u_{i}^{(n-1)}\right| \leq 10^{-5}, \quad\left|\lambda^{(n)}-\lambda^{(n-1)}\right| \leq 10^{-5} .
$$

The values of $\varepsilon$ and $N$, for which we solve the test problem, are $\varepsilon=2^{-i}, i=0,4,8,12,16$; $N=64,128,256,512,1024$. From Tables 1 and 2 we observe that $\varepsilon$-uniform experimental rates of convergence monotonically increase towards one, which is in agreement with the theoretical rate given by Theorem 3.1. 
Table 1 Errors $e_{u}^{\varepsilon, N}$ computed $\varepsilon$-uniform errors $e_{u}^{N}$ and convergence rates $p_{u}^{\varepsilon, N}$ on $\omega_{N}$

\begin{tabular}{llllll}
\hline$\varepsilon$ & $N=64$ & $N=128$ & $N=256$ & $N=512$ & $N=1024$ \\
\hline $2^{0}$ & 0.00464342 & 0.00249526 & 0.00128984 & 0.00065893 & 0.00033245 \\
& 0.896 & 0.952 & 0.969 & 0.987 & \\
$2^{-4}$ & 0.00692191 & 0.00372224 & 0.00192142 & 0.00065782 & 0.00033143 \\
& 0.895 & 0.954 & 0.974 & 0.989 & \\
$2^{-8}$ & 0.00460314 & 0.00247876 & 0.00128398 & 0.00065412 & 0.00032979 \\
& 0.893 & 0.949 & 0.973 & 0.988 & \\
$2^{-12}$ & 0.00460181 & 0.00247290 & 0.00128183 & 0.00065348 & 0.00032947 \\
& 0.896 & 0.948 & 0.972 & 0.988 & \\
$2^{-16}$ & 0.00460557 & 0.00247492 & 0.00128110 & 0.00065175 & 0.00032837 \\
& 0.896 & 0.950 & 0.975 & 0.989 & \\
$e_{u}^{\varepsilon, N}$ & 0.00464342 & 0.00372224 & 0.00128984 & 0.00065893 & 0.00033245 \\
$p_{u}^{\varepsilon_{u}, N}$ & 0.895 & 0.954 & 0.969 & 0.987 & \\
\hline
\end{tabular}

Table 2 Errors $e_{\lambda}^{\varepsilon, N}$ computed $\varepsilon$-uniform errors $e_{\lambda}^{N}$ and convergence rates $p_{\lambda}^{\varepsilon, N}$ on $\omega_{N}$

\begin{tabular}{llllll}
\hline$\varepsilon$ & $N=64$ & $N=128$ & $N=256$ & $N=512$ & $N=1024$ \\
\hline $2^{0}$ & 0.00749478 & 0.00402193 & 0.00204612 & 0.00102448 & 0.00051242 \\
& 0.898 & 0.975 & 0.998 & 1.000 & \\
$2^{-4}$ & 0.00742715 & 0.00399393 & 0.00203893 & 0.00102159 & 0.00051114 \\
& 0.895 & 0.970 & 0.997 & 0.999 & \\
$2^{-8}$ & 0.00723501 & 0.00391767 & 0.00202090 & 0.00101748 & 0.00051051 \\
& 0.885 & 0.955 & 0.990 & 0.995 & \\
$2^{-12}$ & 0.0070768 & 0.00385598 & 0.00200291 & 0.00102333 & 0.00051019 \\
& 0.876 & 0.945 & 0.983 & 0.990 & \\
$2^{-16}$ & 0.00692575 & 0.00379203 & 0.00198065 & 0.00100764 & 0.00050909 \\
& 0.869 & 0.937 & 0.975 & 0.985 & \\
$e_{\lambda}^{\varepsilon, N}$ & 0.00749478 & 0.00402193 & 0.00204612 & 0.00102448 & 0.00313856 \\
$p_{\lambda}^{\varepsilon, N}$ & 0.898 & 0.975 & 0.998 & 1.000 & \\
\hline
\end{tabular}

\section{Conclusion}

We have considered the numerical approximations of a class of quasilinear singularly perturbed first order parameterized differential problems with integral boundary conditions, which serves as the model for many scientific applications. For the numerical solution of this problem, we proposed a uniform convergent finite difference scheme on the graded Bakhvalov mesh. The ideas presented here can be easily applied for solving more complicated initial value problems for parameterized singularly perturbed equations with integral boundary conditions, and the technique presented in the paper can also be applied to high-dimensional systems.

Competing interests

The author declares that they have no competing interests.

Authors' contributions

All authors contributed equally to the manuscript. All authors read and approved the final manuscript.

\section{Publisher's Note}

Springer Nature remains neutral with regard to jurisdictional claims in published maps and institutional affiliations.

Received: 20 December 2017 Accepted: 26 April 2018 Published online: 08 May 2018

\section{References}

1. Farrel, P.A., Hegarty, A.F., Miller, J.J.H., O’Riordan, E., Shishkin, G.l.: Robust Computational Techniques for Boundary Layers. Chapman Hall/CRC, New York (2000)

2. Miller, J.J.H., O'Riordan, E., Shishkin, G.l.: Fitted Numerical Methods for Singular Perturbation Problems. Rev. Ed. World Scientific, Singapore (2012) 
3. O'Malley, R.E.: Singular Perturbation Methods for Ordinary Differential Equations. Springer-Verlag, New York (1991)

4. Roos, H.G., Stynes, M., Tobiska, L.: Numerical Methods for Singularly Perturbed Differential Equations. Springer-Verlag, Berlin (2008)

5. Na, T.Y.: Computational Methods in Engineering Boundary Value Problems. Academic Press, New York (1979)

6. Jankowski, T.: Application of the numerical-analytical method to systems of differential equations with a parameter. Ukr. Math. J. 54(4), 671-683 (2002)

7. Samoilenko, M., Martynyuk, S.V.: Justification of the numerical-analytic method of successive approximations for problems with integral boundary conditions. Ukrain. Mat. Zh. SSR 43, 1231-1239 (1991)

8. Amiraliyeva, I.G., Amiraliyev, G.M.: Uniform difference method for parameterized singularly perturbed delay differential equations. Numer. Algorithms 52, 509-521 (2009)

9. Amiraliyev, G.M., Amiraliyeva, I.G., Kudu, M.: A numerical treatment for singularly perturbed differential equations with integral boundary condition. Appl. Math. Comput. 185, 574-582 (2007)

10. Amiraliyev, G.M., Kudu, M., Duru, H.: Uniform difference method for a parameterized singular perturbation problem. Appl. Math. Comput. 175, 89-100 (2006)

11. Lui, X., Mcare, F.A.: A monotone iterative methods for boundary value problems of parametric differential equation. J. Appl. Math. Stoch. Anal. 14, 183-187 (2001)

12. Pomantale, T: A constructive theorem of existence and uniqueness for the problem $y^{\prime}=f(x, y, \lambda), y(a)=\alpha, y(b)=\beta$. Z. Angew. Math. Mech. 56, 387-388 (1976)

13. Feckan, M.: Parameterized singularly perturbed boundary value problems. J. Math. Anal. Appl. 188, 426-435 (1994)

14. Jankowski, T:: One-step methods for ordinary differential equations with parameters. Apl. Mat. 35(1), 67-83 (1990)

15. Turkyilmazoglu, M.: Analytic approximate solutions of parameterized unperturbed and singularly perturbed boundary value problems. Appl. Math. Model. 35, 3879-3886 (2011)

16. Amiraliyev, G.M., Duru, H.: A note on a parameterized singular perturbation problem. Appl. Math. Comput. 182, 233-242 (2005)

17. Cen, Z: A second order difference scheme for a parameterized singular perturbation problem. J. Comput. Appl. Math. 221, 174-182 (2008)

18. Das, P.: Comparison of a priori and a posteriori meshes for singularly perturbed nonlinear parameterized problems. J. Comput. Appl. Math. 290, 16-25 (2015)

19. Kumar, S., Kumar, M.: A second order uniformly convergent numerical scheme for parameterized singularly perturbed delay differential problems. Numer. Algorithms 76(2), 349-360 (2017)

20. Shakti, D., Mohapatra, J.: Layer-adapted meshes for parameterized singular perturbation problem. Proc. Eng. 127, 539-547 (2015)

21. Xie, F., Wang, J., Zhang, W., He, M.: A novel method for a class of parameterized singularly perturbed boundary value problems. J. Comput. Appl. Math. 213(1), 258-267 (2008)

22. Wang, Y., Chen, S., Wu, X.: A rational spectral collocation method for solving a class of parameterized singular perturbation problems. J. Comput. Appl. Math. 233, 2652-2660 (2004)

23. Cannon, J.R.: The solution of the heat equation subject to the specification of energy. Q. Appl. Math. 21(2), 155-160 (1963)

24. Iokin, N.I: Solution of a boundary value problem in heat conduction theory with nonlocal boundary conditions. Differ. Equ. 13, 294-304 (1977)

25. Nicoud, F., Schönfeld, T.: Integral boundary conditions for unsteady biomedical CFD applications. Int. J. Numer. Methods Fluids 40, 457-465 (2002)

26. Ahmed, B., Khan, R.A., Sivasundaram, S.: Generalizedquasilinearization method for a first order differential equation with integral boundary condition. Dyn. Contin. Discrete Impuls. Syst. 12, 289-296 (2005)

27. Borovykh, N.: Stability in the numerical solution of the heat equation with nonlocal boundary conditions. Appl. Numer. Math. 42, 17-27 (2002)

28. Çakir, M.: A numerical study on the difference solution of singularly perturbed semilinear problem with integral boundary condition. Math. Model. Anal. 21(5), 644-658 (2016)

29. Çakir, M., Amiraliyev, G.M.: Numerical solution of a singularly perturbed three-point boundary value problem. Int. J. Comput. Math. 84(10), 1465-1481 (2007)

30. Cen, Z., Cai, X.: A second-order upwind difference scheme for a singularly perturbed problem with integral boundary condition in neutral network. In: Lecture Notes in Artificial Intelligence, vol. 4693, pp. 175-181. Springer, Berlin (2007)

31. Kudu, M., Amiraliyev, G.M.: Finite difference method for a singularly perturbed differential equations with integral boundary condition. Int. J. Math. Comput. Sci. 24(3), 72-79 (2015)

32. Bakhvalov, N.S.: On the optimization of the methods for solving boundary value problems in the presence of a boundary layer. USSR Comput. Math. Math. Phys. 9(4), 139-166 (1969)

33. Kumar, S., Kumar, M.: Analysis of some numerical methods on layer adapted meshes for singularly perturbed quasilinear systems. Numer. Algorithms 71(1), 139-150 (2016)

34. Amiraliyev, G.M.: The convergence of a finite difference method on layer-adapted mesh for a singularly perturbed system. Appl. Math. Comput. 162(3), 1023-1034 (2005)

35. Şevgin, S.: Numerical solution of a singularly perturbed Volterra integro-differential equation. Adv. Differ. Equ. 2014, Article ID 171 (2014) 